PROCEEDINGS OF THE

AMERICAN MATHEMATICAL SOCIETY

Volume 129, Number 5, Pages 1513-1519

S 0002-9939(00)05632-X

Article electronically published on October 19, 2000

\title{
ON TRANSIENCE OF CARD SHUFFLING
}

\author{
SARA BROFFERIO AND WOLFGANG WOESS
}

(Communicated by Claudia Neuhauser)

\begin{abstract}
We present simple proofs of transience/recurrence for certain card shuffling models, that is, random walks on the infinite symmetric group.
\end{abstract}

\section{CARd ShufFling MOdels}

In this note, we consider several models of shuffling an infinite deck of cards. One of these models has been considered previously by Lawler [La]; our methods (using flows, shorting and comparison of Dirichlet forms) will - partially - simplify his result.

Card shuffling is formalized by performing successive i.i.d. random permutations in the group $S_{\infty}$ of all permutations of the positive integers $\mathbb{N}$ that leave all but finitely many elements fixed. We identify the symmetric group $S_{n}$ with the subgroup of $S_{\infty}$ fixing all elements $>n$, so that $S_{\infty}$ is the union of the $S_{n}$. We read the product of two permutations $x, y$ from left to right, that is, $x \cdot y$ sends $j \in \mathbb{N}$ to $y(x(j))$. Let $\mu$ be a symmetric probability measure on $S_{\infty}$ whose support generates the whole group. Associate with it a sequence $X_{n}, n \geq 1$, of i.i.d. $S_{\infty}$-valued random variables with common distribution $\mu$, and consider the associated random walk $Z_{n}=X_{1} \cdots X_{n}$. This means that we start with the deck of cards in order $\left(Z_{0}\right.$ is the identity), and at each step we choose a random permutation $X_{n}$ according to $\mu$ which tells us how to shuffle once more what we had obtained previously.

The question addressed here is the following. Will the deck of cards eventually return to its original order with probability one (recurrence), or is this probability strictly smaller than 1 (transience)?

We now describe four shuffling models, that is, probabilities $\mu_{1}, \ldots, \mu_{4}$, each one governing another random walk. We start by considering a probability distribution $p(\cdot)$ on $\{2,3, \ldots\}$.

1) First choose $n$ with probability $p(n)$ and then $j \in\{1, \ldots, n-1\}$ with probability $1 /(n-1)$, and exchange the $n$-th with the $j$-th card. Writing $t(n, j)$ for the transposition of $n$ and $j$, we have

$$
\mu_{1}=\sum_{n=2}^{\infty} \sum_{j=1}^{n-1} \frac{p(n)}{n-1} \delta_{t(n, j)} .
$$

Received by the editors March 12, 1999 and, in revised form, July 21, 1999.

2000 Mathematics Subject Classification. Primary 60G50, 60J10; Secondary 60B15.

Key words and phrases. Infinite permutation group, random walk, transience, recurrence.

(C)2000 American Mathematical Society 
2) Choose $n$ with probability $p(n)$, and exchange the $n$-th with the first card. Thus

$$
\mu_{2}=\sum_{n=2}^{\infty} p(n) \delta_{t(n, 1)}
$$

3) First choose $n$ with probability $p(n)$ and then, with probability $1 / 2$ each, either move the $n$-th card to the top or the top card next below to the $n$-th card. Writing $c(n)$ for the cyclic permutation $(1,2, \cdots, n)$ and $c(-n)$ for its inverse, we have

$$
\mu_{3}=\sum_{n=2}^{\infty} \frac{p(n)}{2}\left(\delta_{c(n)}+\delta_{c(-n)}\right) .
$$

4) First choose $n$ with probability $p(n)$ and then $j \in\{1, \ldots, n-1\}$ with probability $1 /(n-1)$, and put the cards in positions $j+1, \ldots, n$ (in the same order) on top of the deck. Thus,

$$
\mu_{4}=\sum_{n=2}^{\infty} \sum_{j=1}^{n-1} \frac{p(n)}{n-1} \delta_{c(n)^{j}} .
$$

Of course, $\delta_{x}$ indicates the Dirac mass at $x \in S_{\infty}$. As a consequence of what we shall prove below, we obtain the following.

Theorem. If $p(n) \sim c /(n-\alpha)$ !, then each of the above random walks is recurrent if $\alpha \leq 2$ and transient if $\alpha>2$.

Here, $\sim$ means that quotients tend to one, $c$ is the proper normalization constant, and for non-integer $\alpha$, the factorial is defined via the Gamma function.

We remark that there is a fifth model, which is much easier but less interesting, namely, when $\mu_{5}$ is equidistributed on each of $S_{n} \backslash S_{n-1}$, with $\mu_{5}\left(S_{n} \backslash S_{n-1}\right)=p(n)$.

Lawler La has considered $\mu_{2}$ (he also assigns positive mass to the identity $i d$, which makes no difference). For proving transience, we have found it easier to start with $\mu_{1}$. We use the method of shorting, which goes back to Nash-Williams [NW] and the flow criterion of Yamasaki [Ya and Lyons [Ly]. We then adapt comparison techniques of Dirichlet forms, that have been successfully applied, for example, to random walks on finite groups by Diaconis and Saloff-Coste [DS], in order to deal with the other three models. We shall need that all $p(n)$ are positive and satisfy certain monotonicity conditions which hold in the special case of the above Theorem.

\section{A GENERAL RECURRENCE CRITERION OF LAWLER}

In the context of model 2, Lawler La has proved a recurrence criterion which adapts with very little additional effort to arbitrary symmetric random walks on direct limits of arbitrary finite groups. For convenience of the reader, we state and prove the general version.

Let $G=\bigcup_{n \geq 1} G_{n}$, where the $G_{n}$ are finite groups and each $G_{n}$ is a (proper) subgroup of $G_{n+1}$. On $G$, consider a symmetric probability measure $\mu$ whose support generates $G$. We may assume that $\mu\left(G_{1}\right)>0$. Again, the random walk is $Z_{n}=X_{1} \cdots X_{n}$, where the $X_{k}$ are i.i.d. $G$-valued with distribution $\mu$. 
Proposition 1. The random walk on $G$ with law $\mu$ is recurrent, if

$$
\sum_{n=1}^{\infty} \frac{1}{\left|G_{n}\right|\left(1-\mu\left(G_{n}\right)\right)}=\infty
$$

Proof. Recurrence is equivalent to $\sum_{n} \mathbb{P}\left[Z_{n}=i d\right]=\infty$, and $\mathbb{P}\left[Z_{n}=i d\right]=\mu^{(n)}(i d)$, where $\mu^{(n)}$ is the $n$-th convolution power. Let $\mu_{k}=\left.\frac{1}{\mu\left(G_{k}\right)} \mu\right|_{G_{k}}$. By symmetry and Cauchy-Schwarz, $\mu_{k}^{(2 n)}(i d) \geq 1 /\left|G_{k}\right|$. Hence, for arbirtrary $k$ and $n$,

$$
\mathbb{P}\left[Z_{2 n}=i d\right] \geq \mathbb{P}\left[Z_{2 n}=i d, X_{i} \in G_{k} \forall i \leq 2 n\right]=\mu_{k}^{(2 n)}(i d) \mu\left(G_{k}\right)^{2 n} \geq \frac{\mu\left(G_{k}\right)^{2 n}}{\left|G_{k}\right|} .
$$

Now let $r(k)=1 /\left(1-\mu\left(G_{k}\right)\right)$. Then $r(k) \leq r(k+1) \rightarrow \infty$. If $2 n \leq r(k)$, then we find

$$
\mathbb{P}\left[Z_{2 n}=i d\right] \geq \frac{1}{\left|G_{k}\right|}\left(1-\frac{1}{r(k)}\right)^{r(k)} \geq \frac{c_{0}}{\left|G_{k}\right|},
$$

where $c_{0}>0$. Next, let $s(k)=\lfloor r(k) / 2\rfloor$. Then

$$
\sum_{n=0}^{\infty} \mathbb{P}\left[Z_{n}=i d\right] \geq \sum_{k=2}^{\infty} \sum_{s(k-1)<n \leq s(k)} \mathbb{P}\left[Z_{2 n}=i d\right] \geq c_{0} \sum_{k=2}^{\infty} \frac{s(k)-s(k-1)}{\left|G_{k}\right|} .
$$

Note that $\left|G_{k+1}\right| \geq 2\left|G_{k}\right|$, whence $2\left|G_{k}\right|^{-1} \geq \sum_{i \geq k}\left|G_{i}\right|^{-1}$. Therefore the right hand term in $(\mathrm{A})$ is

$$
\geq \frac{c_{0}}{2} \sum_{i=2}^{\infty} \sum_{k=2}^{i} \frac{s(k)-s(k-1)}{\left|G_{i}\right|}=\frac{c_{0}}{2} \sum_{i=2}^{\infty} \frac{s(i)-s(1)}{\left|G_{i}\right|} \geq \frac{c_{0}}{2} \sum_{i=2}^{\infty} \frac{s(i)}{\left|G_{i}\right|}-\frac{c_{0} s(1)}{4} .
$$

Now, if $\sum_{n} r(n) /\left|G_{n}\right|=\infty$, then also $\sum_{i} s(i) /\left|G_{i}\right|=\infty$, and the random walk is recurrent.

We remark that special cases of Proposition 1 had been proved previously by Flatto and Pitt $\left[\mathrm{FP}\right.$ (when each $G_{n}$ is cyclic, or when $G$ is the direct sum of finite abelian groups), and before that by Darling and Erdös [DE] (when $G$ is the direct sum of infintely many copies of the two-element group).

\section{UN-SHORTING AND FLOWS}

Returning to our card-shuffling models, we now study transience of $\mu_{1}$.

Proposition 2. The shuffling model no. 1 is transient, provided that

$$
\sum_{n=1}^{\infty} \frac{1}{\left|S_{n}\right| p(n+1)}<\infty
$$

We use well known methods of infinite network theory; see the survey of Woess [Wo]. Let $X$ be an infinite, connected graph with symmetric neighbourhood relation $\sim$. With each edge $[x, y]$, we associate a conductance $c(x, y)=c(y, x)>0$, such that $m(x)=\sum_{y \sim x} c(x, y)<\infty$ for all $x$. The associated reversible Markov chain (random walk) on $X$ has transition probabilities $p(x, y)=c(x, y) / m(x)$. If we start 
with a symmetric transition function $p(\cdot, \cdot)$, then we can set $c(x, y)=p(x, y)$ and $m(x)=1$. For a function $f: X \rightarrow \mathbb{R}$, its Dirichlet sum is

$$
D(f)=\frac{1}{2} \sum_{x \sim y} c(x, y)(f(y)-f(x))^{2} .
$$

The space $\mathcal{D}(X)=\{f: D(f)<\infty\}$ with $\|f\|^{2}=D(f)+f(o)^{2}$ is Hilbert and independent of the choice of the reference vertex $o$. We write $\ell_{0}(X)$ for the finitely supported functions and $\mathcal{D}_{0}(X)$ for their closure in $\mathcal{D}(X)$.

Shorting is the following procedure. Let $X=\bigcup_{i \in \mathcal{I}} X_{i}$ be a partition such that $\mathbf{1}_{X_{i}} \in \mathcal{D}_{0}(X)$ for all $i$. The shorted network is the one over $\mathcal{I}$ where $i \sim j$ if $i \neq j$ and there is some edge between $X_{i}$ and $X_{j}$, with conductances $c^{\prime}(i, j)=$ $\sum_{x \in X_{i}, y \in X_{j}, x \sim y} c(x, y)$. The condition on $\mathbf{1}_{X_{i}}$ guarantees that $m^{\prime}(i)=\sum_{j \sim i} c^{\prime}(i, j)$ $<\infty$. Then one has the following recurrence criterion:

(B) If the random walk on $\mathcal{I}$ is recurrent, then so is the random walk on $X$.

In the case when the shorted network is a half-line, this is Nash-Williams' criterion [NW]. Another well known criterion is the following:

(C) If a network is recurrent then every sub-network is recurrent.

We use these two criteria in the reversed direction. We first construct a locally finite tree with associated conductances given in terms of the $p(n)$ and show that it is transient. We then short it to obtain a sub-network of the one associated with $\mu_{1}$ on $S_{\infty}$.

The vertex set of our tree $T$ is the disjoint union of the $S_{n}$. The root is the identity, and edges occur only between $S_{n-1}$ and $S_{n}$, namely from $x \in S_{n-1}$ to $x \cdot t(n, j)$, where $j<n$, and from $x \in S_{n-1}$ to the copy of $x=x \cdot i d$ in $S_{n}$. We call the latter the improper edges; they are dashed in the figure below.

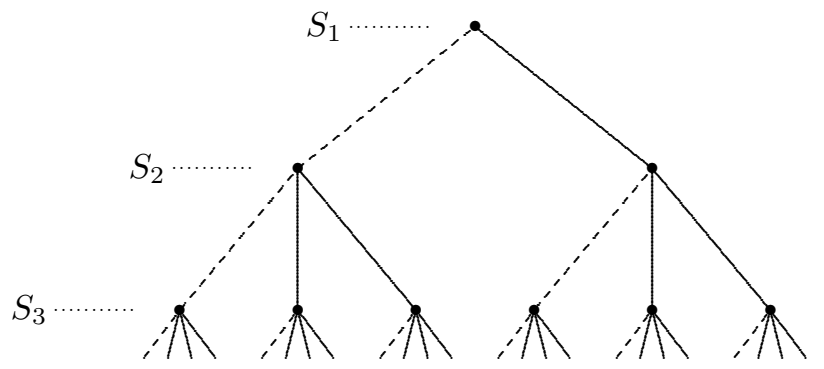

$T$ is indeed a tree because of the fact that for each $y \in S_{n} \backslash S_{n-1}$ there are unique $j<n$ and $x \in S_{n-1}$ such that $y=x \cdot t(n, j)$. To each edge from $S_{n-1}$ to $S_{n}$ in $T$ (proper or improper) we assign conductance $p(n) /(n-1)$.

Proof of Proposition 2. We use flows. On a network $(X, c(\cdot, \cdot))$, a unit flow from $o$ to $\infty$ is a function $F: X \times X$ such that $F(x, y) \neq 0$ only when $x, y$ are neighbours, $F(y, x)=-F(x, y)$, and $\sum_{y: y \sim x} F(x, y)=0$ for all $x \neq o$, while $\sum_{y: y \sim o} F(o, y)=1$. Its energy is $\sum_{x \sim y} F(x, y)^{2} / c(x, y)$. The flow criterion of Yamasaki [Ya] and Lyons $\mathrm{Ly}$ is the following.

The random walk on $X$ is transient if and only if there is a (unit) flow from $o$ to $\infty$ with finite energy.

We now use the unit flow from $o=i d \in S_{1}$ that branches equally at each vertex when moving down from $o$. That is, $F(x, y)=1 / n$ ! when $x \in S_{n-1}$ and $y \in S_{n}$ are neighbours in $T$ (properly or improperly). It is an immediate exercise to verify 
that $F$ has finite energy precisely when the condition of Proposition 2 holds, thus proving transience of the random walk on $T$.

If $x \in S_{\infty}$, then $x \in S_{k}$ for a minimal $k=k(x)$, and in $T$, there is a ray (halfline) $R_{x}$ of successive improper edges starting at $x$ (the latter viewed as a vertex in $\left.S_{k(x)}\right)$. We now short each of these improper rays to a single point, again denoted $x$. Let $R_{x}(n)$ be the finite path obtained by truncating $R_{x}$ at level $n \geq k(x)$. Then $D\left(\mathbf{1}_{R_{x}}-\mathbf{1}_{R_{x}(n)}\right)=\frac{p(n)}{n-1}+\sum_{j>n} p(j) \rightarrow 0$, whence the indicator function of each improper ray is in $\mathcal{D}_{0}(T)$, and shorting is legitimate. By (B), the random walk on the shorted network is also transient. This is in fact a spanning tree of the Cayley graph of $S_{\infty}$ with respect to the generating set consisting of all transpositions, and it is certainly a subnetwork of the network corresponding to the random walk with law $\mu_{1}$. Our tree $T$ arises from un-shorting this subnetwork. By $(\mathrm{C})$, the latter must also be transient.

We remark that writing down this proof takes more time than explaining it with the figure at hand. In particular, the three ingredients (flows, shorting, passing to sub-networks) have by now become part of the random walk folklore. The critical eye might observe that we could have constructed the finite energy flow directly on the shorted tree. We think that the above approach is nicer. Also, it seems that "un-shorting" has not been used before for proving transience.

\section{Comparison of Dirichlet forms}

In order to deduce from Proposition 2 transience criteria for models 2, 3 and 4, we use the following method. Let $X$ be equipped with two different graph structures, associated conductances and corresponding transition matrices $P$ and $Q$. We write $D_{P}$ and $D_{Q}$ for the respective Dirichlet sums.

(D) If $D_{Q}(f) \leq c D_{P}(f)$ for all $f \in \ell_{0}(X)$, where $c>0$, then transience of $Q$ implies transience of $P$.

Let us adapt this to the context of a group $G$. If $a \in G$, then we write

$$
D_{a}(f)=\sum_{x \in G}(f(x \cdot a)-f(x))^{2},
$$

and if $\mu$ is a symmetric probability measure on $G$, then $D_{\mu}(f)=\sum_{a \in G} \mu(a) D_{a}(f)$. The graph structure associated with $\mu$ is the Cayley graph of $G$ with respect to $S=\operatorname{supp}(\mu)$. Note that $D_{\mu}$ is not precisely the corresponding Dirichlet sum as defined in $\S 2$, because edges $[x, x \cdot a]$ are counted twice when $a \in S$ is not idempotent. This does not matter when applying (D), as the factor $1 / 2$ which is eventually missing can be subsumed in the constant $c$. The basic tool is the following.

Lemma. If $a=b_{1} \cdots b_{k}$, then $D_{a}(f) \leq k\left(D_{b_{1}}(f)+\cdots+D_{b_{k}}(f)\right)$.

Proof. This is straightforward via the Cauchy-Schwarz inequality and the expansion $f(x \cdot a)-f(x)=\sum_{i=1}^{k}\left(f\left(x \cdot y_{i}\right)-f\left(x \cdot y_{i-1}\right)\right)$, where $y_{i}=b_{1} \cdots b_{i}$.

Proposition 3. If $p(n)>0$ for all $n \geq 2$ and $\lim \sup p(n+1) / p(n)<1$, then there are $c_{2}, c_{4}>0$ such that $D_{\mu_{1}} \leq c_{2} D_{\mu_{2}}$ and $D_{\mu_{1}} \leq c_{4} D_{\mu_{4}}$. 
Proof. Model 2. If $2 \leq j \leq n-1$, then $t(n, j)=t(j, 1) \cdot t(n, 1) \cdot t(j, 1)$, and the Lemma yields $D_{t(n, j)} \leq 6 D_{t(j, 1)}+3 D_{t(n, 1)}$. Therefore

$$
\begin{aligned}
D_{\mu_{1}} & \leq \sum_{n=2}^{\infty} \frac{p(n)}{n-1} D_{t(n, 1)}+\sum_{n=3}^{\infty} \sum_{j=2}^{n-1}\left(6 \frac{p(n)}{n-1} D_{t(j, 1)}+3 \frac{p(n)}{n-1} D_{t(n, 1)}\right) \\
& \leq 6 \sum_{n=2}^{\infty}\left(p(n)+\sum_{k=n+1}^{\infty} \frac{p(k)}{k-1}\right) D_{t(n, 1)} .
\end{aligned}
$$

The assumptions on $p(n)$ yield that $\frac{p(n+k)}{n+k-1} \leq C \lambda^{k} \frac{p(n)}{n-1}$ for all $n, k$, where $C \geq 1$ and $0<\lambda<1$. Therefore

$$
\sum_{k=n+1}^{\infty} \frac{p(k)}{k-1} \leq \bar{C} \frac{p(n)}{n-1}
$$

with $\bar{C}=C \lambda /(1-\lambda)$, and we find $c_{2}=1+\bar{C}$.

Model 4. We have $t(n, j)=c(-n)^{j} \cdot c(n-1)^{j} \cdot c(-j)$ when $n>j \geq 2$, while $t(n, 1)=c(-n) \cdot c(n-1)$ for $n \geq 3$, and $t(2,1)=c(2)$. Observe that $D_{a^{-1}}(f)=$ $D_{a}(f)$. Therefore

$$
\begin{aligned}
& D_{\mu_{1}} \leq p(2) D_{c(2)}+2 \sum_{n=3}^{\infty} \frac{p(n)}{n-1}\left(D_{c(n)}+D_{c(n-1)}\right) \\
&+3 \sum_{n=3}^{\infty} \sum_{j=2}^{n-1} \frac{p(n)}{n-1}\left(D_{c(n)^{j}}+D_{c(n-1)^{j}}+D_{c(j)}\right) \\
&=\left(p(2)+p(3)+3 \sum_{k=3}^{\infty} \frac{p(k)}{k-1}\right) D_{c(2)} \\
&+\sum_{n=3}^{\infty}\left(2 \frac{p(n)}{n-1}+2 \frac{p(n+1)}{n}+3 \sum_{k=n+1}^{\infty} \frac{p(k)}{k-1}\right) D_{c(n)} \\
&+\sum_{n=3}^{\infty} \sum_{j=2}^{n-1}\left(3 \frac{p(n)}{n-1}+3 \frac{p(n+1)}{n}\right) D_{c(n)^{j}} .
\end{aligned}
$$

Once more using (E), we find $c_{4}=3+3 C /(1-\lambda)$.

Proposition 4. If $p(2)>0$, then there is $c_{3}>0$ such that $D_{\mu_{2}} \leq c_{3} D_{\mu_{3}}$.

Proof. We have $t(2,1)=c(2)$. If $n \geq 3$, then $t(n, 1)=c(n) \cdot c(2) \cdot c(-n)$. Therefore

$$
\begin{aligned}
D_{\mu_{2}} & \leq p(2) D_{c(2)}+\sum_{n=3}^{\infty}\left(6 p(n) D_{c(n)}+3 p(n) D_{c(2)}\right) \\
& =(3-2 p(2)) D_{c(2)}+6 \sum_{n=3}^{\infty} p(n) D_{c(n)},
\end{aligned}
$$

which is bounded above by $\max \{6,3 / p(2)\} D_{\mu_{3}}$.

Combining Propositions 2-4 with criterion (D), we now obtain the following.

Corollary. If $\sum_{n=1}^{\infty} 1 /\left(\left|S_{n}\right| p(n+1)\right)<\infty$ and $\lim \sup p(n+1) / p(n)<1$, then the shuffling models no. 2-4 are transient. 
In particular, when $\lim \sup p(n+1) / p(n)<1$, then the condition of Proposition 2 is necessary and sufficient for transience. The Theorem stated in the introduction arises as a special case.

In Proposition 3 and the Corollary, one can of course replace the condition $\lim \sup p(n+1) / p(n)<1$ by some weaker hypothesis that imples (E), or by (E) itself.

Lawler La] has proved that for transience of model no. 2, already the first of the two conditions in the above Corollary is sufficient. In view of Proposition 4, the limsup-condition can also be dropped when considering transience of model no. 3 .

We conclude with an outlook. If one wants to reduce the gap between the criteria of Propositions 1 and 2, one possible approach will be to use the representation theory of $S_{\infty}$, or the $S_{n}$, respectively. Denote by $\sigma_{n}$ the uniform distribution on $\{t(n, j): j<n\}$. Then the $\sigma_{n}$ commute under convolution, which is why model 1 is preferable to the others in this context, compare with Diaconis [Di]. We intend to come back to this in future work.

\section{REFERENCES}

[DE] D. A. Darling and P. Erdös, On the recurrence of a certain chain, Proc. Amer. Math. Soc. 19 (1968), 336-338. MR 36:6012

[Di] P. Diaconis, Group representations in Probability and Statistics, IMS, Hayward, California, 1988. MR 90a:60001

[DS] P. Diaconis and L. Saloff-Coste, Comparison techniques for random walk on finite groups, Ann. Probab. 21 (1993), 2131-2156. [MR 95a:60009]

[FP] L. Flatto and J. Pitt, Recurrence criteria for random walks on countable abelian groups, Illinois J. Math. 18 (1974), 1-19. MR 49:6363

[La] G. F. Lawler, Recurrence and transience for a card shuffing model, Comb. Probab. Comp. 4 (1995), 133-142. MR 96g:60085

[Ly] T. Lyons, A simple criterion for transience of a reversible Markov chain, Ann. Probab. 11 (1983), 393-402. MR 84e:60102

[NW] C. St. J. A. Nash-Williams, Random walks and electric currents in networks, Proc. Cambridge Phil. Soc. 55 (1959), 181-194. MR 23:A2239

[Wo] W. Woess, Random walks on infinite graphs and groups: a survey on selected topics, Bull. London Math. Soc. 26 (1994), 1-60. MR 94i:60081

[Ya] M. Yamasaki, Discrete potentials on an infinite network, Mem. Fac. Sci. Shimane Univ. 13 (1979), 31-44. MR 81h:31016

Laboratoire de Probabilités, Université de Paris 6, 4 Place Jussieu, 75252 Paris, FRANCE

E-mail address: brofferi@ccr.jussieu.fr

Dipartimento di Matematica e Applicazioni, Università di Milano "Bicocca", Via BicocCA DEgli Arcimboldi 8, 20126 Milano, Italia

Current address, W. Woess: Institut für Mathematik C, Technische Universität Graz, A-8010 Graz, Austria

E-mail address: woess@weyl.math.tu-graz.ac.at

$U R L:$ http://www.cis.tu-graz.ac.at/mathc/woess 